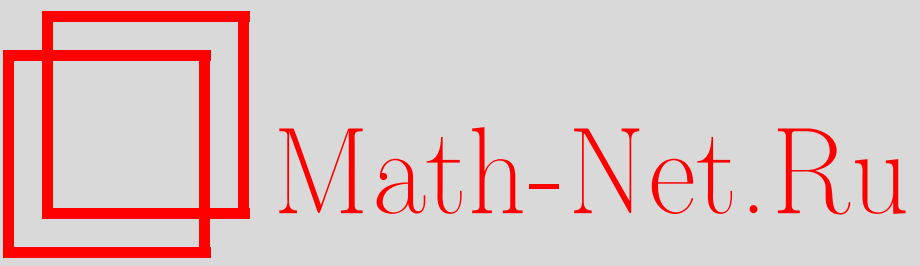

Ю. И. Шевченко, Е. В. Скрыдлова, Плоскостное пространство проективной связности, Итоги науки и техн. Сер. Соврем. мат. и ее прил. Темат. обз., 2020, том 180, 113-119

DOI: https://doi.org/10.36535/0233-6723-2020-180-113-119

Использование Общероссийского математического портала Math-Net.Ru подразумевает, что вы прочитали и согласны с пользовательским соглашением

http: //www . mathnet.ru/rus/agreement

Параметры загрузки:

IP : 54.198 .64 .247

26 апреля 2023 г., 18:25:09 


\title{
ПЛОСКОСТНОЕ ПРОСТРАНСТВО ПРОЕКТИВНОЙ СВЯЗНОСТИ
}

\author{
(c) 2020 г. Ю. И. ШЕВЧЕНКО, Е. В. СКРЫДЛОВА
}

\begin{abstract}
АннотАция. Проективное пространство, в котором неэффективно действует линейная группа, позволяет построить соответствующее пространство проективной связности Картана. Показано, что структурные уравнения пространства Картана дают возможность получить дифференциальные уравнения для компонент тензора проективной кривизны-кручения. Этот тензор содержит тензор кручения, расширенный тензор кручения и тензор аффинной кривизны-кручения. Найден аналог тождеств Бианки. Сформулирован допускающий обобщение алгоритм построения структурных уравнений пространства проективной связности Картана. С помощью обобщенного алгоритма построены структурные уравнения плоскостного пространства проективной связности, частными случаями которого являются линейчатое пространство проективной связности Акивиса, точечное пространство проективной связности Картана и двойственное ему гиперплоскостное пространство проективной связности. Доказано, что тензор кривизны-кручения плоскостного пространства проективной связности имеет три подтензора, один из которых - аналог тензора кручения пространства Картана.
\end{abstract}

Ключевые слова: пространство проективной связности Картана, тензор кривизны-кручения, аналог тождеств Бианки, линейчатое пространство проективной связности, плоскостное пространство проективной связности.

\section{PLANAR SPACE WITH PROJECTIVE CONNECTION}

\author{
(c) 2020 YU. I. SHEVCHENKO, E. V. SKRYDLOVA
}

\begin{abstract}
A projective space in which a linear group acts ineffectively allows one to construct the corresponding space of Cartan projective connection. We show that the structure equations of the Cartan space allow one to obtain differential equations for the components of the projective curvaturetorsion tensor. This tensor contains the torsion tensor, the extended torsion tensor, and the affine curvature-torsion tensor. An analog of the Bianchi identities is found. A generalizable algorithm for constructing structure equations of the space of Cartan projective connection is formulated. Using a generalized algorithm, we construct the structure equations of the planar space of projective connection, whose special cases are the ruled space of Akivis projective connection, point space of Cartan projective connection, and its dual hyperplanar space of projective connection. We also prove that the curvaturetorsion tensor of a plane space with projective connection has three subtensors, one of which is an analog of the torsion tensor of the Cartan space.
\end{abstract}

Keywords and phrases: space of Cartan projective connection, curvature-torsion tensor, analog of Bianchi identities, ruled space of projective connection, planar space of projective connection.

AMS Subject Classification: 53B10

1. Пространство проективной связности Картана. Рассмотрим $n$-мерное проективное пространство $P_{n}$, отнесенное к однородному подвижному реперу $\left\{A_{I^{\prime}}\right\}$ с деривационными формулами

$$
d A_{I^{\prime}}=\theta_{I^{\prime}}^{J^{\prime}} A_{J^{\prime}}, \quad I^{\prime}, J^{\prime}, \ldots=\overline{0, n}
$$


причем линейные дифференциальные формы $\theta_{I^{\prime}}^{J^{\prime}}$ удовлетворяют структурным уравнениям линейной группы GL $(n+1)$ :

$$
d \theta_{J^{\prime}}^{I^{\prime}}=\theta_{J^{\prime}}^{K^{\prime}} \wedge \theta_{K^{\prime}}^{I^{\prime}}
$$

Эти уравнения выражают полную интегрируемость дифференциальных уравнений (1).

Группа $\mathrm{GL}(n+1)$ действует в проективном пространстве $P_{n}$ неэффективно. От этого избавляются двумя способами:

(1) берут специальную линейную группу SGL $(n+1)$, выделяемую условием $\theta_{I^{\prime}}^{I^{\prime}}=0$, тогда имеем эффективное проективное пространство;

(2) используют проективную группу $\mathrm{GP}(n)$ - фактор-группу с $n(n+2)$ структурными формами $\theta_{0}^{I}, \theta_{J}^{I}-\delta_{J}^{I} \theta_{0}^{0}, \theta_{I}^{0}, I, J, \ldots=\overline{1, n}$; тогда можно говорить о фактор-эффективном проективном пространстве.

Будем рассматривать неэффективное проективное пространство $P_{n}$ и соответствующие пространства проективной связности.

В пространстве $P_{n}$ зафиксируем точку $A$, превратив его в центропроективное пространство $P_{n}^{0}$. Совместим вершину $A_{0}$ репера $\left\{A_{0}, A_{I}\right\}$ с центром $A$-пространства $P_{n}^{0}$. Формулы (1) и уравнения (2) запишем подробнее:

$$
\begin{aligned}
d A_{0} & =\theta_{0}^{0} A_{0}+\theta_{0}^{I} A_{I}, \\
d A_{I} & =\theta_{I}^{0} A_{0}+\theta_{I}^{J} A_{J}, \\
d \theta_{0}^{I} & =\theta_{0}^{J} \wedge\left(\theta_{J}^{I}-\delta_{J}^{I} \theta_{0}^{0}\right), \\
d \theta_{J}^{I} & =\theta_{J}^{0} \wedge \theta_{0}^{I}+\theta_{J}^{K} \wedge \theta_{K}^{I}, \\
d \theta_{I}^{0} & =\theta_{I}^{0} \wedge \theta_{0}^{0}+\theta_{I}^{J} \wedge \theta_{J}^{0}, \\
d \theta_{0}^{0} & =\theta_{0}^{I} \wedge \theta_{I}^{0} .
\end{aligned}
$$

Из формулы (3) следуют уравнения стационарности точки $A_{0}$ :

$$
\theta_{0}^{I}=0 .
$$

В силу структурных уравнений (5) эта система уравнений вполне интегрируема. Она выделяет из линейной группы $\mathrm{GL}(n+1)$ центролинейную подгруппу стационарности $G_{n^{2}+n+1}$ со структурными уравнениями

$$
d \vartheta_{J}^{I}=\vartheta_{J}^{K} \wedge \vartheta_{K}^{I}, \quad d \vartheta_{I}^{0}=\vartheta_{I}^{0} \wedge \vartheta_{0}^{0}+\vartheta_{I}^{J} \wedge \vartheta_{J}^{0}, \quad d \vartheta_{0}^{0}=0 \quad\left(\vartheta=\left.\theta\right|_{(5)}\right) .
$$

Возьмем $n$-мерное гладкое многообразие $M_{n}$. Для любой точки $x \in M_{n}$ имеем деривационную формулу Акивиса (см. [2, с. 54]):

$$
d x=\omega^{I} e_{I},
$$

где векторы $e_{I}$ образуют базис касательного пространства $T_{n}(x)$ к многообразию $M_{n}$ в точке $x$. Базисные формы $\omega^{I}$ удовлетворяют структурным уравнениям Лаптева (см. [7]):

$$
d \omega^{I}=\omega^{J} \wedge \Omega_{J}^{I}
$$

которые показывают полную интегрируемость системы уравнений

$$
\omega^{I}=0,
$$

фиксирующей точку $x=x_{0}$.

K каждой точке $x$ многообразия $M_{n}$ присоединим проективное пространство $P_{n}(x)$; тогда для семейства пространств $P_{n}(x)$ обобщения структурных уравнений $(2)$ будут иметь следующий вид:

$$
d \omega_{J^{\prime}}^{I^{\prime}}=\omega_{J^{\prime}}^{K^{\prime}} \wedge \omega_{K^{\prime}}^{I^{\prime}}+\omega^{K} \wedge \omega_{J^{\prime} K}^{I^{\prime}},
$$

причем

$$
\bar{\omega}_{J^{\prime}}^{I^{\prime}}=\theta_{J^{\prime}}^{I^{\prime}} \quad\left(\bar{\omega}=\left.\omega\right|_{(13)}\right) .
$$


Действительно, уравнения (13)-(15) позволяют выделить линейную группу $\mathrm{GL}(n+1)$ со структурными уравнениями (2), действующую в фиксированном проективном пространстве $P_{n}\left(x_{0}\right)$. Более того, из условия (15) следует $\bar{\omega}_{0}^{I}=\theta_{0}^{I}$, поэтому при отождествлении

$$
\omega_{0}^{I}=\omega^{I}
$$

в пространстве $P_{n}\left(x_{0}\right)$ выполняются равенства (9). Значит, фиксированное присоединяемое пространство $P_{n}\left(x_{0}\right)$ является центропроективным пространством $P_{n}^{0}\left(x_{0}\right)$ с центром $x_{0}=A_{0}$. В этом случае будем говорить о приклеивании (ср. [3, с. 109]) проективных пространств $P_{n}(x)$ к базе $M_{n}$, в результате чего они становятся центропроективными пространствами $P_{n}^{0}(x)$.

Картан показал (см. [4]), что смежное пространство $P_{n}^{0}(x+d x)$ отображается на исходное пространство $P_{n}^{0}(x)$, если формы $\omega_{J^{\prime} K}^{I^{\prime}}$ в структурных уравнениях (14) являются линейными комбинациями базисных форм $\omega_{0}^{I}$ :

$$
\omega_{J^{\prime} K}^{I^{\prime}}=R_{J^{\prime} K L}^{I^{\prime}} \omega_{0}^{L}
$$

Тогда уравнения (14) становятся структурными уравнениями пространства проективной связности Картана $P_{n, n}$ :

$$
d \omega_{J^{\prime}}^{I^{\prime}}=\omega_{J^{\prime}}^{K^{\prime}} \wedge \omega_{K^{\prime}}^{I^{\prime}}+R_{J^{\prime} K L}^{I^{\prime}} \omega_{0}^{K} \wedge \omega_{0}^{L} .
$$

Антикоммутативность внешних произведений линейных форм объясняет антисимметрию коэффициентов:

$$
R_{J^{\prime}(K L)}^{I^{\prime}}=0
$$

где круглые скобки обозначают симметрирование.

Из уравнений (17) следует, что базисные формы $\omega_{0}^{I}$ удовлетворяют структурным уравнениям

$$
d \omega_{0}^{I}=\omega_{0}^{J} \wedge\left(\omega_{J}^{I}-\delta_{J}^{I} \omega_{0}^{0}+R_{0 J K}^{I} \omega_{0}^{K}\right) .
$$

Сопоставляя их с уравнениями (12), убеждаемся в возможности отождествления (16) при

$$
\Omega_{J}^{I}=\omega_{J}^{I}-\delta_{J}^{I} \omega_{0}^{0}+R_{0 J K}^{I} \omega_{0}^{K} .
$$

Если выполнится вполне интегрируемая в силу (19) система уравнений

$$
\omega_{0}^{I}=0,
$$

то уравнения (17) превратятся в структурные уравнения (10) центролинейной группы $G_{n^{2}+n+1}$.

Утверждение 1. Пространство проективной связности Картана $P_{n, n}$ со структурными уравнениями (17) (ср. [9]) есть главное расслоение $G_{n^{2}+n+1}\left(M_{n}\right)$ над базой $M_{n}$ - гладким многообразием со структурными уравнениями (19), типовым слоем которого служит центролинейная группа $G_{n^{2}+n+1} \subset \mathrm{GL}(n+1)$, действующая неэффективно в каждом центропроективном пространстве $P_{n}^{0}(x)$, приклеенном к базе в точке $x \in M_{n}$.

Утверждение 2. Пространство $P_{n, n}$ является особым пространством фундаментально-групповой связности Лаптева (см. [6], так как базисные формы $\omega_{0}^{I}$ входят в состав форм связности $\omega_{J^{\prime}}^{I^{\prime}}$, поэтому пространство $P_{n, n}$, рассматриваемое как $G_{n^{2}+n+1}\left(M_{n}\right)$, не является главным расслоением со связностью (см., например, [5, с. 167]).

2. Тензор кривизны-кручения и его подтензоры. Структурные уравнения (17) пространства проективной связности Картана $P_{n, n}$ позволяют найти дифференциальные уравнения для компонент тензора кривизны-кручения $R_{J^{\prime} K L}^{I^{\prime}}$. Продифференцируем уравнения (17) внешним образом:

$$
\nabla R_{J^{\prime} K L}^{I^{\prime}} \wedge \omega_{0}^{K} \wedge \omega_{0}^{L}+2 R_{J^{\prime} K L}^{I^{\prime}} R_{0 M N}^{K} \omega_{0}^{L} \wedge \omega_{0}^{M} \wedge \omega_{0}^{N}=0,
$$

где ковариантный дифференциал $\nabla$ от компонент $R_{J^{\prime} K L}^{I^{\prime}}$ относительно проективной связности имеет следующий вид:

$$
\nabla R_{J^{\prime} K L}^{I^{\prime}}=\Delta R_{J^{\prime} K L}^{I^{\prime}}+2 R_{J^{\prime} K L}^{I^{\prime}} \omega_{0}^{0}
$$

а дифференциальный оператор $\Delta$ действует по формуле

$$
\Delta R_{J^{\prime} K L}^{I^{\prime}}=d R_{J^{\prime} K L}^{I^{\prime}}+R_{J^{\prime} K L}^{M^{\prime}} \omega_{M^{\prime}}^{I^{\prime}}-R_{M^{\prime} K L}^{I^{\prime}} \omega_{J^{\prime}}^{M^{\prime}}-R_{J^{\prime} M L}^{I^{\prime}} \omega_{K}^{M}-R_{J^{\prime} K M}^{I^{\prime}} \omega_{L}^{M} .
$$


Представим кубичные уравнения (20) в виде

$$
\left(\nabla R_{J^{\prime} K L}^{I^{\prime}} \wedge \omega_{0}^{L}+2 R_{J^{\prime} N L}^{I^{\prime}} R_{0 M K}^{N} \omega_{0}^{M} \wedge \omega_{0}^{L}\right) \wedge \omega_{0}^{K}=0 .
$$

Разрешим их по лемме Лаптева (см. [7]):

$$
\nabla R_{J^{\prime} K L}^{I^{\prime}} \wedge \omega_{0}^{L}+2 R_{J^{\prime} N L}^{I^{\prime}} R_{0 M K}^{N} \omega_{0}^{M} \wedge \omega_{0}^{L}=\omega_{0}^{L} \wedge \omega_{J^{\prime} K L}^{I^{\prime}},
$$

причем выполняются условия полуголономности (см. [10]):

$$
\begin{gathered}
\omega_{J^{\prime} K L}^{I^{\prime}} \wedge \omega_{0}^{K} \wedge \omega_{0}^{L}=0 \Longleftrightarrow \omega_{J^{\prime}[K L]}^{I^{\prime}} \wedge \omega_{0}^{K} \wedge \omega_{0}^{L}=0 \Longleftrightarrow \omega_{J^{\prime}[K L]}^{I^{\prime}}=\lambda_{J^{\prime} K L M}^{I^{\prime}} \omega_{0}^{M}, \\
\lambda_{J^{\prime}(K L) M}^{I^{\prime}}=0, \\
\lambda_{J^{\prime}\{K L M\}}^{I^{\prime}}=0,
\end{gathered}
$$

где квадратные скобки обозначают альтернирование, а фигурные скобки - циклирование.

Преобразуем квадратичные уравнения (22):

$$
\left(\nabla R_{J^{\prime} K L}^{I^{\prime}}+2 R_{J^{\prime} N L}^{I^{\prime}} R_{0 M K}^{N} \omega_{0}^{M}+\omega_{J^{\prime} K L}^{I^{\prime}}\right) \wedge \omega_{0}^{L}=0 .
$$

Разрешим эти уравнения по лемме Картана и проальтернируем результат по индексам $K, L$ с учетом условий антисимметрии (18):

$$
\nabla R_{J^{\prime} K L}^{I^{\prime}}+2 R_{J^{\prime} N[L}^{I^{\prime}} R_{|0 M| K]}^{N} \omega_{0}^{M}+\omega_{J^{\prime}[K L]}^{I^{\prime}}=R_{J^{\prime}[K L] M}^{I^{\prime}} \omega_{0}^{M}
$$

причем $R_{J^{\prime} K[L M]}^{I^{\prime}}=0$. С помощью условия полуголономности (23) дифференциальные уравнения (26) примут окончательный вид

$$
\nabla R_{J^{\prime} K L}^{I^{\prime}}=R_{J^{\prime} K L ; M}^{I^{\prime}} \omega_{0}^{M}
$$

где

$$
R_{J^{\prime} K L ; M}^{I^{\prime}}=R_{J^{\prime}[K L] M}^{I^{\prime}}-2 R_{J^{\prime} N[L}^{I^{\prime}} R_{|0 M| K]}^{N}-\lambda_{J^{\prime} K L M}^{I^{\prime}}
$$

Отсюда в силу антисимметрии (24) следует, что ковариантные производные $R_{J^{\prime} K L ; M}^{I^{\prime}}$ компонент $R_{J^{\prime} K L}^{I^{\prime}}$ антисимметричны по двум индексам:

$$
R_{J^{\prime}(K L) ; M}^{I^{\prime}}=0 .
$$

Используя равенства (21), запишем подробнее дифференциальные уравнения (27) в виде сравнений по модулю базисных форм $\omega_{0}^{J}$ :

$$
\begin{gathered}
\Delta R_{0 K L}^{I}+R_{0 K L}^{I} \omega_{0}^{0} \cong 0 \quad\left(\bmod \omega_{0}^{J}\right), \\
\Delta R_{J K L}^{I}+2 R_{J K L}^{I} \omega_{0}^{0}-R_{0 K L}^{I} \omega_{J}^{0} \cong 0, \\
\Delta R_{0 K L}^{0}+2 R_{0 K L}^{0} \omega_{0}^{0}+R_{0 K L}^{I} \omega_{I}^{0} \cong 0, \\
\Delta R_{J K L}^{0}+3 R_{J K L}^{0} \omega_{0}^{0}+R_{J K L}^{I} \omega_{I}^{0}-R_{0 K L}^{0} \omega_{J}^{0} \cong 0 .
\end{gathered}
$$

Теорема 1. Структурные уравнения (17) пространства проективной связности Картана $P_{n, n}$ содержат компоненты тензора проективной кривизнь-кручения $R_{J^{\prime} K L}^{I^{\prime}}$, удовлетворяющие дифференцилљным уравнениям (27). Дифференииальные сравнения (29)-(32)) показывают, что тензор $R_{J^{\prime} K L}^{I^{\prime}}$ имеет три подтензора: $R_{0 K L}^{I}-$ тензор кручения, $\left\{R_{0 K L}^{0}, R_{0 K L}^{I}\right\}-$ расширенный тензор кручения, $\left\{R_{J K L}^{I}, R_{0 K L}^{I}\right\}-$ тензор афббнной кривизны-кручения.

При аннулировании тензора кручения: $R_{0 K L}^{I}=0$ дифференциальные сравнения (32) не изменятся, а сравнения (30)-(31) упростятся:

$$
\Delta R_{J K L}^{I}+2 R_{J K L}^{I} \omega_{0}^{0} \cong 0, \quad \Delta R_{0 K L}^{0}+2 R_{0 K L}^{0} \omega_{0}^{0} \cong 0 .
$$

Следствие. Компоненты тензора кривизны $\left\{R_{J K L}^{I}, R_{0 K L}^{0}, R_{J K L}^{0}\right\}$ пространства проектияной связности Картана без кручения $P_{n, n}^{0}$ удовлетворяют дифференииальным сравнениям (32), (33), поэтому тензор кривизны имеет два подтензора: $R_{0 K L}^{0}$ u $R_{J K L}^{I}-$ аналог тензора кривизнъ афбинной связности. 
Замечание. Если тензор проективной кривизны-кручения обратится в нуль: $R_{J^{\prime} K L}^{I^{\prime}}=0$, то уравнения (17) примут вид

$$
d \omega_{J^{\prime}}^{I^{\prime}}=\omega_{J^{\prime}}^{K^{\prime}} \wedge \omega_{K^{\prime}}^{I^{\prime}}
$$

совпадающие со структурными уравнениями (2) линейной группы $\mathrm{GL}(n+1)$. Значит, пространство проективной связности Картана $P_{n, n}$ обобщает линейную группу $\mathrm{GL}(n+1)$, действующую неэффективно в проективном пространстве $P_{n}$.

3. Аналог тождеств Бианки. Кубичные уравнения (20) дают возможность не только найти дифференциальные уравнения (27) компонент тензора кривизны-кручения, но и получить аналог тождеств Бианки. Из уравнений (20), (27) следует

$$
\left(R_{J^{\prime} K L ; M}^{I^{\prime}}+2 R_{J^{\prime} N L}^{I^{\prime}} R_{0 M K}^{N}\right) \omega_{0}^{K} \wedge \omega_{0}^{L} \wedge \omega_{0}^{M}=0
$$

откуда получим

$$
R_{J^{\prime}[K L ; M]}^{I^{\prime}}+2 R_{J^{\prime} N[L}^{I^{\prime}} R_{|0| M K]}^{N}=0 .
$$

При альтернировании по трем индексам, используя условия (18), (28) - антисимметрию по двум индексам, перейдем к циклированию:

$$
R_{J^{\prime}\{K L ; M\}}^{I^{\prime}}+2 R_{J^{\prime} N\{K}^{I^{\prime}} R_{|0| L M\}}^{N}=0 .
$$

Утверждение 3. Компоненты тензора кривизны-кручения $R_{J^{\prime} K L}^{I^{\prime}}$ и их ковариантные производные $R_{J^{\prime} K L ; M}^{I^{\prime}}$ относительно проективной связности Картана удовлетворяют аналогу тождеств Бианки (34).

4. Построение структурных уравнений пространств проективной связности. Отталкиваясь от структурных уравнений $(2)$ линейной группы GL $(n+1)$, действующую неэффективно в проективном пространстве $P_{n}$, сформулируем алгоритм построения структурных уравнений (17) пространства проективной связности Картана $P_{n, n}$.

А. Относим проективное пространство $P_{n}$ к однородному реперу $\left\{A_{I^{\prime}}\right\}$. В пространстве $P_{n}$ неэффективно действует линейная группа $\mathrm{GL}(n+1)$ со структурными уравнениями $(2)$.

В. Берем точку $A_{0} \in P_{n}$ и находим уравнения ее стационарности. Левые части этих уравнений содержат $n$ структурных форм группы GL $(n+1)$, которые называются базисными формами.

C. Структурные уравнения (17) пространства $P_{n, n}$ получаются в результате обобщения уравнений (2) путем добавления линейных комбинаций внешних произведений аналогов базисных форм с коэффициентами - компонентами тензора кривизны-кручения.

Назовем точку $A_{0}$ фигурой приклеивания, а пространство $P_{n, n}$ - точечным пространством проективной связности. В обозначении Картана пространства проективной связности $P_{n, n}$ первое $n$ будем считать размерностью проективного пространства, $n=\operatorname{dim} P_{n}$, а второе $n-$ размерностью базы, равной размерности пространства фигур приклеивания в пространстве $P_{n}$.

Алгоритм построения структурных уравнений пространства $P_{n, n}$ легко распространяется на другие пространства проективной связности, в которых в качестве фигуры приклеивания берется не точка, а любая линейная фигура (см. [8]). Например, гиперплоскостное пространство проективной связности $P_{n, n}^{*}$, двойственное пространству $P_{n, n}$, имеет следующие структурные уравнения:

$$
d \omega_{J^{\prime}}^{I^{\prime}}=\omega_{J^{\prime}}^{K^{\prime}} \wedge \omega_{K^{\prime}}^{I^{\prime}}+R_{J^{\prime}}^{I^{\prime} K L} \omega_{K}^{0} \wedge \omega_{L}^{0}, \quad R_{J^{\prime}}^{I^{\prime}(K L)}=0 .
$$

5. Пространство плоскостной проективной связности. Введем новое пространство проективной связности. В качестве фигуры приклеивания возьмем $m$-мерную плоскость $P_{m} \subset P_{n}$ $(0 \leqslant m \leqslant n-1)$. Разобьем значения штрихованных индексов на две серии:

$$
I^{\prime}=(\alpha, i) ; \quad \alpha, \ldots=\overline{0, m} ; \quad i, \ldots=\overline{m+1, n} .
$$

Поместим вершины $A_{\alpha}$ подвижного репера $\left\{A_{\alpha}, A_{i}\right\}$ на плоскость $P_{m}$. Из деривационных формул (1) следует

$$
d A_{\alpha}=\theta_{\alpha}^{\beta} A_{\beta}+\theta_{\alpha}^{i} A_{i}
$$


откуда получаем уравнения стационарности плоскости $P_{m}$ :

$$
\theta_{\alpha}^{i}=0 .
$$

Значит, $\theta_{\alpha}^{i}$ - базисные формы, число которых равно $(m+1)(n-m)$. Структурные уравнения $(2)$ дают уравнения

$$
d \theta_{\alpha}^{i}=\theta_{\beta}^{j} \wedge\left(\delta_{\alpha}^{\beta} \theta_{j}^{i}-\delta_{j}^{i} \theta_{\alpha}^{\beta}\right),
$$

которые обеспечивают полную интегрируемость системы уравнений (36).

Применяя алгоритм из п. 4 в случае плоскости приклеивания $P_{m}$, построим структурные уравнения плоскостного пространства проективной связности $P_{n,(m+1)(n-m)}$ :

$$
d \omega_{J^{\prime}}^{I^{\prime}}=\omega_{J^{\prime}}^{K^{\prime}} \wedge \omega_{K^{\prime}}^{I^{\prime}}+R_{J^{\prime} i j}^{I^{\prime} \alpha \beta} \omega_{\alpha}^{i} \wedge \omega_{\beta}^{j},
$$

причем выполняются условия антисимметрии по парам индексов:

$$
R_{J^{\prime}}^{I^{\prime}}\left(\begin{array}{c}
\alpha \beta \\
i j
\end{array}\right)=0 .
$$

Выделим три частных случая пространства $P_{n,(m+1)(n-m)}$ :

(1) $m=0$ - пространство проективной связности Картана $P_{n, n}$ со структурными уравнениями (17),

(2) $m=1$ - линейчатое пространство проективной связности Акивиса $P_{n, 2(n-1)}$ (см. [1]),

(3) $m=n-1$ - гиперплоскостное пространство проективной связности $P_{n, n}^{*}$ со структурными уравнениями (35).

Замкнем структурные уравнения (28):

$$
\nabla R_{J^{\prime} i j}^{I^{\prime} \alpha \beta} \wedge \omega_{\alpha}^{i} \wedge \omega_{\beta}^{j}+2 R_{J^{\prime} i j}^{I^{\prime} \alpha \beta} R_{\alpha k l}^{i \gamma \delta} \omega_{\beta}^{j} \wedge \omega_{\gamma}^{k} \wedge \omega_{\delta}^{l}=0,
$$

где ковариантный дифференциал $\nabla$ действует следующим образом:

$$
\nabla R_{J^{\prime} i j}^{I^{\prime} \alpha \beta}=d R_{J^{\prime} i j}^{I^{\prime} \alpha \beta}+R_{J^{\prime} i j}^{K^{\prime} \alpha \beta} \omega_{K^{\prime}}^{I^{\prime}}+R_{J^{\prime} i j}^{I^{\prime} \gamma \beta} \omega_{\gamma}^{\alpha}+R_{J^{\prime} i j}^{I^{\prime} \alpha \gamma} \omega_{\gamma}^{\beta}-R_{K^{\prime} i j}^{I^{\prime} \alpha \beta} \omega_{J^{\prime}}^{K^{\prime}}-R_{J^{\prime} k j}^{I^{\prime} \alpha \beta} \omega_{i}^{k}-R_{J^{\prime} i k}^{I^{\prime} \alpha \beta} \omega_{j}^{k} .
$$

Кубичные уравнения (39) позволяют найти дифференциальные уравнения

$$
\nabla R_{J^{\prime} i j}^{I^{\prime} \alpha \beta}=R_{J^{\prime} i j}^{I^{\prime} \alpha \beta} ;_{k}^{\gamma} \omega_{\gamma}^{k},
$$

причем из условий антисимметрии (38) следует

$$
R_{J^{\prime}}^{I^{\prime}}\left(\begin{array}{c}
\alpha \beta \\
i j
\end{array}\right) ; ;_{k}^{\gamma}=0 .
$$

Уравнения (39), (40) дают:

$$
\left(R_{J^{\prime} i j}^{I^{\prime} \alpha \beta} ; k+2 R_{J^{\prime} l j}^{I^{\prime} \delta \beta} R_{\delta k i}^{l \gamma \alpha}\right) \omega_{\alpha}^{i} \wedge \omega_{\beta}^{j} \wedge \omega_{\gamma}^{k}=0,
$$

откуда получим

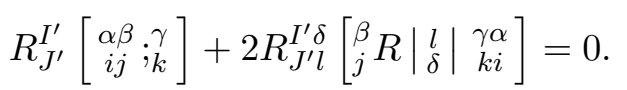

Преобразуем эти равенства с помощью условий антисимметрии (38), (41):

$$
\left.R_{J^{\prime}}^{I^{\prime}}\left\{\begin{array}{c}
\alpha \beta \\
i j
\end{array} ; \underset{k}{\gamma}\right\}\right\}+2 R_{J^{\prime} l}^{I^{\prime} \delta}\left\{\left.\begin{array}{c}
\alpha \\
i
\end{array} R\right|_{\delta} ^{l} \mid \begin{array}{c}
\beta \gamma \\
j k
\end{array}\right\}=0
$$

Запишем дифференциальные уравнения (40) в виде подобных сравнений:

$$
\begin{gathered}
\nabla R_{\gamma i j}^{k \alpha \beta} \cong 0 \quad\left(\bmod \omega_{\alpha}^{i}\right), \quad \nabla R_{\delta i j}^{\gamma \alpha \beta}-R_{\delta i j}^{k \alpha \beta} \omega_{k}^{\gamma} \cong 0, \\
\nabla R_{k i j}^{l \alpha \beta}+R_{\gamma i j}^{l \alpha \beta} \omega_{k}^{\gamma} \cong 0, \quad \nabla R_{k i j}^{\gamma \alpha \beta}+R_{\delta i j}^{\gamma \alpha \beta} \omega_{k}^{\delta}-R_{k i j}^{l \alpha \beta} \omega_{l}^{\gamma} \cong 0 .
\end{gathered}
$$

Теорема 2. Пространство плоскостной проективной связности $P_{n,(m+1)(n-m)}$ определяется структурными уравнениями (37), которые содержат компоненты аналога тензора кривизны-кручения $R_{J^{\prime} i j}^{I^{\prime} \alpha}$, удовлетворяющие дифференциальным уравнениям (40). Эти компоненты вместе с их ковариантными производными $R_{J^{\prime} i j}^{I^{\prime} \alpha \beta} ; \underset{k}{\gamma}$ относительно плоскостной проективной 
связности подчиняются аналогу тождеств Бианки (42). Подробные дифференциальные сравнения (43) для компонент тензора $R_{J^{\prime} i j}^{I^{\prime} \alpha \beta}$ показывают, что он имеет три подтензора: $\left\{R_{\delta i j}^{\gamma \alpha \beta}, R_{0}\right\}$, $\left\{R_{k i j}^{l \alpha \beta}, R_{0}\right\}, R_{0}=\left\{R_{\gamma i j}^{k \alpha \beta}\right\}$-аналог тензора кручения.

\section{СПИСОК ЛИТЕРАТУРЫ}

1. Акивис M. А. Об изоклинных три-тканях и их интерпретации в линейчатом пространстве проективной связности// Сиб. мат. ж. - 1974. - 15, № 1. - С. 3-15.

2. Аживис М. А. Многомерная дифференциальная геометрия. - Калинин, 1977.

3. Евтушик Л. Е., Лумисте Ю. Г., Остиану Н. М., Широков А. П. Дифференциально-геометрические структуры на многообразиях// Пробл. геом. - 1979. - 9. - С. 3-248.

4. Картан Э. Пространство аффинной, проективной и конформной связности. - Из-во Казан. ун-та, 1962.

5. Кобаяси Ш. Группы преобразований в дифференциальной геометрии. - М., 1986.

6. Лаптев Г. Ф. Дифференциальная геометрия погруженных многообразий// Тр. Моск. мат. о-ва. 1953. - 2. - C. $275-382$.

7. Лаптев $Г$. Ф. Основные инфинитезимальные структуры высших порядков на гладком многообразии// Тр. геом. семин. ВИНИТИ. - 1966. - 1. - С. 139-189.

8. Шевченко Ю. И. Структура оснащения многообразия линейных фигур// Тез. докл. VI Прибалт. геом. конф.. - Таллин, 1984. - С. 136-137.

9. Шевченко Ю. И. Центропроективная связность в пространстве проективной связности Картана// Дифф. геом. многообр. фигур. - 2005. - 36. - С. 154-160.

10. Шевченко Ю. И. Голономные и полуголономные подмногообразия гладких многообразий// Дифф. геом. многообр. фигур. - 2015. - 46. - С. 168-177.

Шевченко Юрий Иванович

Балтийский федеральный университет им. И. Канта, Калининград

E-mail: IUSHevchenko@kantiana.ru

Скрыдлова Елена Викторовна

Балтийский федеральный университет им. И. Канта, Калининград

E-mail: ESkrydlova@kantiana.ru 